\title{
GÉNERO Y REALEZA EN EL ANTIGUO EGIPTO, DEL DINÁSTICO TEMPRANO AL IMPERIO NUEVO
}

\author{
Gender and Reyalty in Ancient Egypt, since Early Dynastic to New Empire
}

\author{
Alba BRAVO YONTE \\ Departamento de Prehistoria, Historia Antigua y Arqueología \\ Universidad de Salamanca
}

Fecha de recepción: 02-02-2010

Fecha de aceptación: 05-02-2010

\begin{abstract}
RESUMEN: La historiografía tradicional ha tenido hasta hace relativamente poco al varón como único protagonista, pero, desde 1960, se han desarrollado estudios, en diversos campos, para devolver a las mujeres su historia, y a la historia la otra mitad de la humanidad, omitida e invisibilizada, voluntaria o involuntariamente. En este caso concreto pretendemos plantear una investigación, a través de diversos tipos de fuentes, sobre el papel económico, social, político y religioso de las mujeres en la realeza del Antiguo Egipto, desde el Dinástico Temprano (c. 3000- 2686 a.n.e.) al Imperio Nuevo (c. 1550-1069 a.n.e.), por medio de la comparación de casos concretos, estudio de nombres y titulaturas, relaciones familiares, principios de la realeza egipcia, etc. De este modo, intentaremos comprobar si la hipótesis de un sistema dual de realeza existió verdaderamente. La metodología a seguir será el materialismo histórico, junto a métodos y técnicas de la metodología de género y herramientas propias de la antropología sociológica.
\end{abstract}

Palabras Clave: historiografía tradicional, historia de las mujeres, Antiguo Egipto, sistema dual de realeza, metodología de género.

ABSTRACT: The traditional historiography has had, until a few time ago, the man as only and main character, but, since 1960, some studies have been developed, at several fields, to return her history to the women, and to the history the other part of the humanity, omitted and made invisible, voluntarily or involuntarily. In this case we pretend to set out a research about the economic, social, politic and religious role of the women in the royalty of 
Ancient Egypt, through diverse types of sources, since the Early dynastic (c. 3000- 2686 a.n.e.) to the New Empire (c. 1550-1069 a.n.e.). All of this would be made comparing some cases, studying the names and titularities, family relationships, principles of Egyptian royalty, etc. In this way, we try to probe if my hypothesis of a dual concept of royalty was true. The methodology to follow will be the historic materialism, joint to the methods and techniques of gender methodology and social anthropology owner tools.

Keywords: Traditional historiography, Women history, Ancient Egypt, Dual system of royalty, Gender methodology.

\section{INTRODUCCIÓN}

La civilización egipcia ha suscitado el interés de los europeos, de forma casi continuada, y sobre ella han escrito multitud de autores, como Hecateo de Mileto (s. VI a.n.e.) o Manetón (siglo III a.n.e.), entre otros.

A finales del s. XVI y principios del XVII comenzaron las primeras expediciones a Egipto en busca de antigüedades, misiones que no pueden ser calificadas de arqueológicas propiamente. Un ejemplo de ellas es el viaje de Pietro della Valle (1586-1652), que regresó a Italia con algunas momias y con importantes manuscritos coptos, por lo que se empezó a prestar atención al estudio de esta lengua; ello constituirá la base para el posterior desciframiento de la escritura jeroglífica. El polígrafo Athanasius Kircher (16021680) fue uno de los primeros que intentó descifrar dicha escritura, aunque erró en su punto de partida al suponer que la escritura egipcia era silábica.

A pesar de que fueron muchos los esfuerzos previos, la expedición napoleónica ${ }^{1}$ de 1798 fue el momento clave, debido al hallazgo de la "Piedra de Rosetta" y al estudio y registro de todos los aspectos del Egipto antiguo y moderno por el equipo de eruditos que acompañaban a la campaña napoleónica. Fue, finalmente, François Champollion (1790-1832) quien logró descifrar la escritura jeroglífica, en torno a 1822-24, marcando este hecho el inicio de la Egiptología como disciplina independiente.

A mediados del s. XIX, fueron los trabajos de campo en territorio egipcio los que adquirieron mayor importancia gracias a la labor de Auguste Mariette (1821-1881), que excavó en numerosos lugares y fundó, en 1858, el Museo Egipcio y el Servicio de Antigüedades ${ }^{2}$. Posteriormente, será sir Flinders Petrie (1853-1942) quien sobresalga por su arduo trabajo en el descubrimiento de hallazgos de diversas épocas, aunque sus métodos y técnicas de excavación, superados posteriormente por G. A. Reisner (1867-1942), no permitieron documentar de forma estrictamente científica los hallazgos.

En el s. XX las excavaciones y estudios se centraron en el Valle de los Reyes, en Tebas, pero, además, se excavaron y analizaron otros cementerios 
reales, como el de Gizeh, con hallazgos tan destacados como el de la tumba de Hetep-heres $\mathrm{I}^{3}$, las ruinas urbanas de Tell el-Amarna, el poblado obrero de Deir el-Medina y su necrópolis adyacente, etc. Se recopilaron y publicaron los monumentos existentes, completando así la labor de la excavación. También en Nubia se han realizado excavaciones y estudios exhaustivos, sobre todo desde la construcción y posteriores ampliaciones de la presa de Asuán, con descubrimientos espectaculares y las consiguientes campañas de salva-

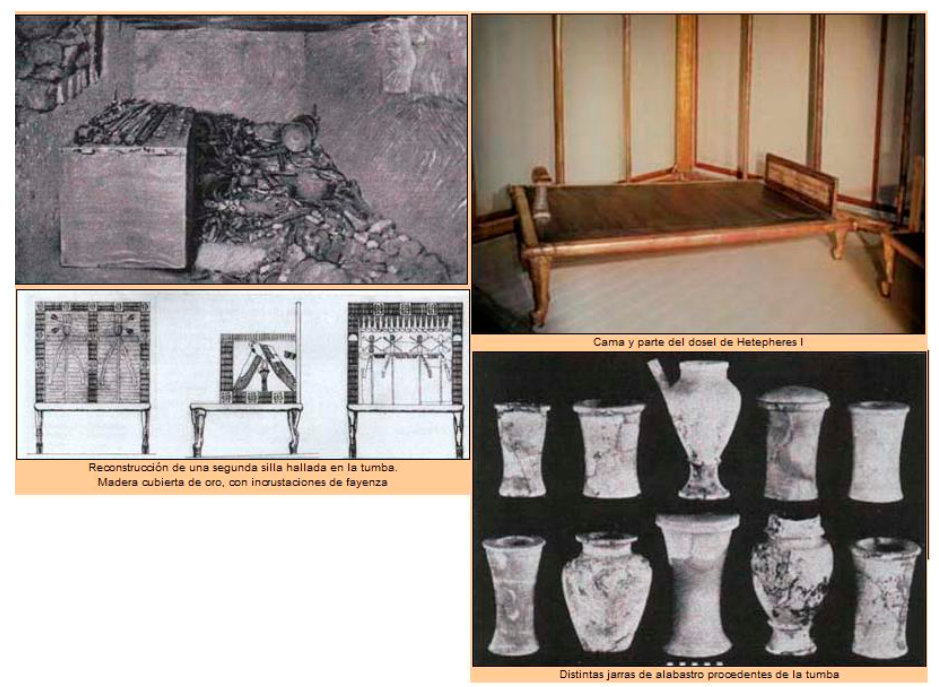

Figura I: Tumba de Hetep-heres I (madre de Keops), descubierta por Reisner en Gizeh, en 1925. Extraída de: http://www.egiptomania.com/arte/tumbas/guiza/hetepheres/ (acceso: $16 / 01 / 2010)$.

ción de monumentos nubios.

El objetivo primordial de los egiptólogos fue siempre la comprensión de la lengua. A comienzos del s. XX se lograron importantes avances en el conocimiento del demótico, la escritura cursiva y la lengua de los períodos tardío y grecorromano (por F. L. Griffith y W. Spiegelberg). Por su parte, sir Alan Gardiner (1879-1963) publicó una gramática del lenguaje egipcio del Imperio Medio (c. 2055-1650 a.n.e.), obra fundamental, y H. J. Polotsky (1905-1991) un estudio revolucionario sobre la gramática egipcia y copta. Otros estudios que merecen destacarse, pues han transformado áreas enteras dentro de la Egiptología, son los realizados por Heinrich Schäfer (1868-1957), que publicó una obra fundamental sobre las representaciones de la escultura y los bajorrelieves; Gerhard Fecht demostró que la mayoría de los textos egipcios fueron escritos en una métrica 
de tipo acentual; y Hermann Kees y Henri Frankfort se centraron en la investigación e interpretación de la religión egipcia.

"Todo cuanto se ha hecho hasta ahora en la egiptología no es más que el preludio de lo que queda por llegar”, según John Baines y Jaromír Malek Hoy día es una disciplina académica convencional, ascendiendo a más de veinte países en los que se encuentra representada ${ }^{5}$. Son ya numerosos los egiptólogos de profesión cuyos estudios han abarcado temáticas tan diversas como la lengua, la literatura ${ }^{6}$, la música, la religión, la mitología, el Estado egipcio y su estructura (la realeza, la administración, la sociedad, la esclavitud, la economía, la educación...), la medicina, las matemáticas, la astronomía, el arte, etc.

Pero, aunque sobre la vida y los logros de los faraones se han escrito innumerables obras, sus madres, esposas e hijas han recibido una atención bastante limitada, salvo casos concretos como Hatshepsut, Nefertiti, Nefertari o Cleopatra (estudios, sobre todo, a partir del Reino Nuevo). La razón estriba en que todos los estudios estuvieron siempre marcados por la perspectiva androcéntrica occidental, invisibilizando la historia de las mujeres, que "aunque no es independiente de la de los hombres, es una historia propia"7. Fue hacia 1960 cuando surgió el concepto género "como una categoría fundamental de la realidad social, cultural e histórica, y de la percepción y el estudio de dicha realidad". Además, es "un criterio normativo (...), a través del cual las personas construyen su identidad, según ciertas pautas que conducen (...) a ubicarse como varón o como mujer con todas sus consecuencias e implicaciones" $"$ Al concepto de género se une el de patriarcado, para completar y precisar en mayor medida el anterior, que implica la noción de poder y jerarquización de las relaciones. Así, el género aportó, por tanto, la llamada "otra voz" hecho hasta ahora y transformarlo, cambiando con ello la sociedad y la cultura de la época. Esto condujo a la aparición de los denominados Estudios de mujeres. De esta forma cobra importancia "restituir las mujeres a la historia" y, por tanto, "restituir la historia de las mujeres"11. Asimismo, "los esfuerzos por unir la historia de una mitad con la otra, y éstas con la historia en general, han dado un paso crucial al concebir a las mujeres como un grupo sociocultural"12.

Con relación a la aparición de la historia de las mujeres y de la historia de género, en las investigaciones en el campo de la Egiptología, se pueden destacar trabajos sobre cuestiones generales como el de J. Vercoutter, "La femme et l'Egypte ancienne" (1965); Gay Robins, Women in ancient Egypt (1993); Ch. Desroches Noblecourt, La mujer en tiempos de los faraones (1999); Zahi Hawass, Silent Images: Women in Pharaonic Egypt (2000) o Lana Troy, "Good and bad Women" (1984), entre otros. Pero, también, podemos encontrar estudios más específicos como Marriage and Matrimonial property in 
Ancient Egypt, de P. W. Pestman (1961); Essays on Femenine Titles of the Middle Kingdom and Related Subjects, de W. A. Ward (1986); Egyptian Women of the Old Kingdom and of the Heracleopolitan Period, de Henry G. Fischer (1989); Patterns of Queenship in Ancient Egyptian Myth and History, de Lana Troy (1986); Women and Religion in Ancient Egypt, de Barbara S. Lesko (2002); sin olvidar a Deborah Sweeney y su estudio de la correspondencia femenina, Correspondence and Dialogue: Pragmatic Features in Late Ramesside Letter-writing (2001) o a Roger S. Bagnall y Raffaella Cribiore y sus Women's letters from ancient Egypt : $300 B C-A D$ 800 (2006), por destacar algunos ${ }^{13}$.

\section{OBjetivos}

En lo que se refiere al Trabajo Fin de Máster que he de realizar, y posteriormente en la Tesis Doctoral, pretendo colaborar a esclarecer la historia de las mujeres, en concreto en el Antiguo Egipto, pues es esencial para que más adelante se pueda realizar una historia de género. Si falta el estudio de la historia de una mitad de la humanidad no es posible comparar la de los dos géneros (según M. Perrot), y, por lo tanto, de los tipos de roles sociales y relaciones que se dan entre y dentro de ellos en cada época y lugar. La historia general, hasta hace relativamente poco, ha sido específica del varón, como sujeto y objeto universal del discurso historiográfico, pero "la conciencia de la alteridad, de la diferencia, de la desigualdad entre la historia femenina y la masculina ha venido a complementarse con una conciencia y un estudio histórico de la alteridad, de las diferencias, y de las desigualdades entre las propias mujeres"14. En este sentido, Gisela Bock plantea la realización de una historia sólo de mujeres, en primer lugar, que aborde todo dominio social:

los ámbitos en los que sólo tienen presencia las mujeres (...), aquellos en los que las mijeres resultan ser mayoría (...), aquellos en los que igualan en número a los hombres (...), aquellos en los que las mujeres son una minoría en relación a los hombres (como el trabajo fabril y el de la historiografía) y, finalmente, aquellos en los que están ausentes en su conjunto (...). Es decir, la historia de las mujeres sólo puede ser comprendida en plural, no en singular, pero su variedad existe en el contexto de la compleja historia de la totalidad del sexo femenino ${ }^{15}$.

De este modo, el trabajo general de investigación, en el que se enmarca el Trabajo Fin de Máster, como inicio de la labor, se realizará mediante la aplicación de una perspectiva crítica, analizando los trabajos anteriores que no tuvieron en cuenta a esa "otra" mitad de la población, a veces, tan sobresaliente en la civilización egipcia, pudiendo extraer los logros y deficiencias de esas investigaciones, analizando lo que se contó, a veces, y de lo que se 
trataba en realidad, tras un nuevo estudio, desde otro punto de vista, de esas cuestiones. Ya que, como se va confirmando, la situación estaba bastante equiparada entre hombres y mujeres, hasta la llegada de los griegos (s. II a.n.e.) y de los romanos, después (s. I a.n.e.). Existen evidencias de que las mujeres podían desempeñar cargos de relevancia, administrar sus bienes, heredar como cualquier otro hijo (aunque, bien es cierto que, en el Antiguo Egipto, la herencia era libre y ser descendiente no garantizaba nada $)^{16}$, elegir con quien se casaban (ya que en Egipto el matrimonio es un acto privado entendido como convivir bajo el mismo techo), trabajar en un amplio abanico de actividades, participar en ritos y ceremonias religiosas, tener una tumba con fórmulas mágicas que le guiaran en su camino al más allá17, etc.

El punto clave de mi investigación se basa en el hecho de que, revisando la documentación antigua y la bibliografía contemporánea, no existe ninguna cláusula o mención que impida a las mujeres gobernar, de la misma forma que los hombres. Por el contrario, se puede observar en la documentación religiosa y mitológica la continua presencia de parejas de dioses, en complementariedad, equilibrio e interdependencia de ambos sexos. A modo de ejemplo, encontramos la pareja de dioses Isis- Osiris (hijos de Geb y Nut), donde se puede ver cómo se complementan. Él es el primer gobernante de Egipto, que será descuartizado por Seth (su hermano). Isis lo buscará y resucitará, con ayuda de Anubis, y gobernará hasta que el sucesor, Horus, tenga edad para tal cometido. Aunque su nombre significa trono, esto no quiere decir que su relevancia se reduzca a ser mero asiento del rey, sino que porta un símbolo de poder de vital importancia para la realeza, al igual que las coronas o el cetro real. Cada uno (Isis y Osiris) posee sus atributos y sus propias funciones, para lograr el equilibrio, el orden (Maat), sin ser unas de mayor valor que otras. Relacionando estos datos religiosos y mitológicos con la institución de la realeza, y sabiendo que la religión y la mitología son reflejo de la mentalidad de una sociedad, cabría preguntarse si la realeza se regía por el mismo principio de complementariedad y necesidad mutua de ambos elementos, femenino y masculino (ya que, como comúnmente se dice, los faraones, al ser representantes de las divinidades en el gobierno del país, debían actuar de forma análoga a éstas), y, si es así, ¿las mujeres podían reinar al igual que los hombres?

Partiendo de estas anotaciones, los aspectos significativos a investigar serían los siguientes. Como punto central se estudiará el papel social, económico y político de la realeza (nzw.t, término neutro), en el Antiguo Egipto, para un mayor conocimiento de la misma, y poder, así, comprobar si la hipótesis que planteo, de un sistema dual de realeza, existió realmente, $\mathrm{y}$, dentro del mismo, analizar el papel de las mujeres. El papel de la reina siempre ha 
sido minusvalorado y visto desde una perspectiva androcéntrica, por lo que cabe un mayor interés de estudio.

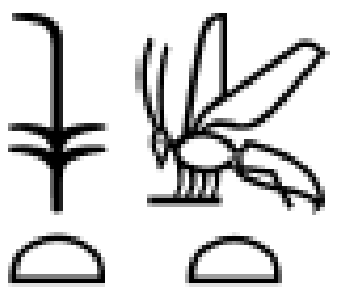

Figura II: Jeroglífico de la realeza (no de rey, como tradicionalmente se ha interpretado). Los semicírculos de la parte inferior se transcriben como .t, partícula de los nombres neutros (en otros casos de los femeninos).

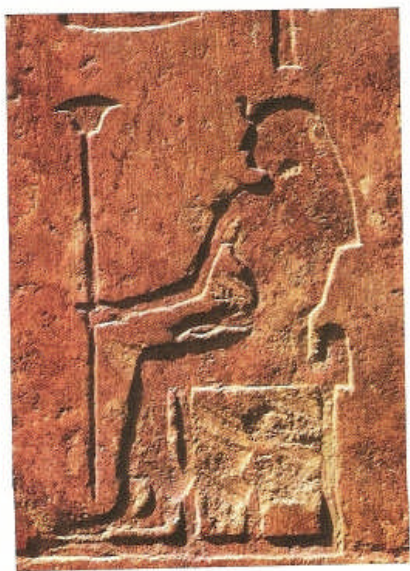

Figura III: Relieve de la reina Khent-kaus II, de su tumba en Abusir. BEDMAN, T., Reinas de Egipto. El secreto del poder, Madrid: Alianza Ed., 2007, p. 46.

Asimismo, cabría profundizar en los elementos y relaciones claves en el seno de la familia real. Y, a través de la prosopografía e inscripciones biográficas, estudiadas y analizadas críticamente, confrontar los momentos en los que las mujeres tuvieron una presencia clave en la familia real.

En relación con todo ello, se examinará el papel de la diosa Neith ${ }^{18}$ en la configuración de la monarquía, puesto que algunas reinas incorporan en la composición de su nombre el de Neith, como, por ejemplo, Mer-Neith, en la Dinastía I, o bien hacen uso del símbolo de dicha diosa, como la reina Hetep-heres I, que lo introduce en el repertorio iconográfico de su mobiliario (cf. figs. I y IV). 

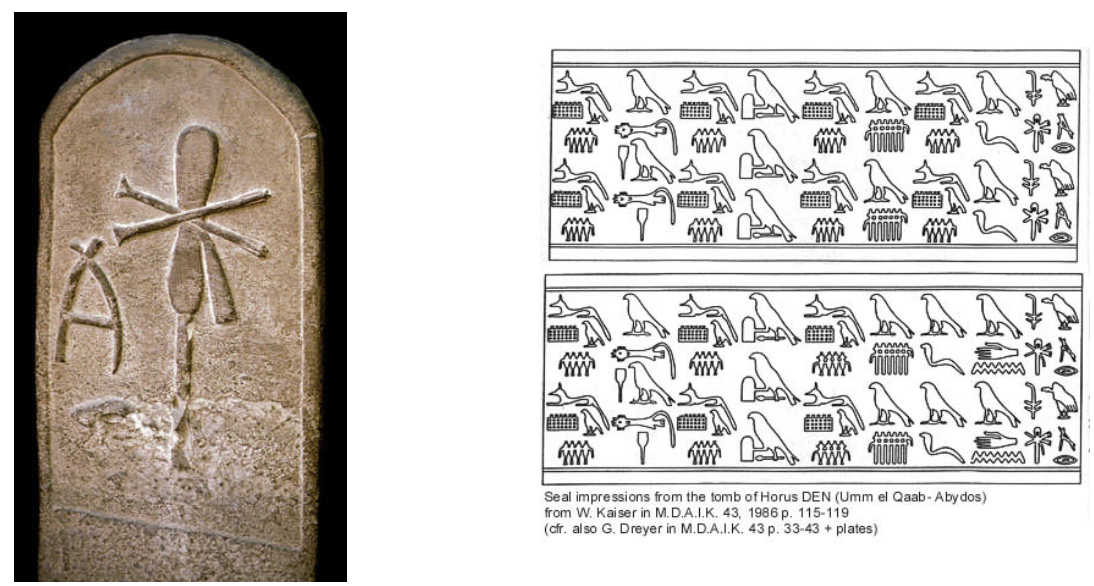

Figuras IV-V: Estela Mer-neith, Abydos; Impresiones reales de la tumba de Den, Abydos, donde aparece el nombre de Merneith (extraídas de: http:/ /xoomer.virgilio.it/francescoraf/hesyra/merneith.html acceso 19/01/2010)

De igual manera, se analizarán los principios de la realeza egipcia y cómo se organizaba, desde el Dinástico temprano (c. 3000- 2686 a.n.e. ${ }^{19}$ al Imperio Nuevo (c. 1550-1069 a.n.e.), revisando casos concretos y mostrando su evolución por comparación de personajes significativos de distintas épocas, incluyendo el caso de Cleopatra, como último exponente de la cultura faraónica.

También, se examinarán los nombres de las reinas y princesas y sus significados, así como su titulatura.

Del mismo modo, se estudiarán los casos en los que el papel importante en la sociedad se trasladó al ámbito teológico, como en el caso de las "Adoratrices" o "Esposas del Dios" (a partir de la XVIII dinastía), que, en definitiva, suponía poder político paras las reinas y princesas.

De esta forma, se intentará recopilar toda la información disponible, a través de diversas fuentes, sobre las reinas y princesas del Antiguo Egipto, desentrañando las relaciones interpersonales y explorando el papel que desempeñaron en el ámbito económico, social, político y religioso, así como se podrá comprobar, en el transcurso de la investigación, si la hipótesis que planteo es correcta o no. 


\section{Metodología y FUENTES}

Para todo investigador que inicia su bagaje en el arduo camino de la investigación no resulta fácil saber claramente, desde un principio, qué metodología de investigación seguirá. Sin embargo, de las diversas tendencias habidas para la Historia Antigua, como el historicismo, el positivismo, el materialismo histórico, la Escuela de Annales y el postmodernismo, en este caso resultará más adecuado el empleo del materialismo histórico, como método analítico, que distingue una base socio-económica, formada por fuerzas productivas y relaciones de producción, y una "superestructura" que abarca aspectos políticos, jurídicos e ideológicos. Algunos egiptólogos recomiendan también el empleo de herramientas propias de la Antropología sociológica.

Asimismo, para el tema que interesa tratar, habría que añadir los métodos y técnicas inherentes a los estudios de género. Resulta obligado, inicialmente, revelar la historia de las mujeres, para después realizar una historia de género. En este sentido, lo novedoso de la historia de las mujeres no son los métodos, sino las cuestiones que plantea y las relaciones que establece. Ninguna historia es totalmente neutral, aunque se pretenda, pues depende de decisiones previamente tomadas que dan sentido a la investigación. Por su parte, la historia de género nos ha ofrecido algunas aportaciones metodológicas de gran utilidad para el análisis histórico como la relectura de las fuentes, nuevos modelos interpretativos y nuevas categorías analíticas (como género, subordinación, dominación, masculinidad-feminidad, igualdad-desigualdaddiferencia, etc.)

No obstante, debe señalarse que para el oficio del historiador, y en concreto en la Egiptología, la interdisciplinariedad es fundamental para una buena comprensión del pasado. De ahí la colaboración de unos años aquí de diversas disciplinas con respecto a la historia, denominadas, comúnmente, "auxiliares": geografía, cronología, arqueología, historia del arte, epigrafía, papirología, prosopografía, filología, paleopatología, criptografía, etc.

En suma, “el 'método histórico' requiere una etapa, la heurística, de búsqueda de información o fuentes sobre la que se va a asentar el estudio, la crítica con su argumentación que vendrá a dar fuerza a la elaboración del trabajo, formulando hipótesis o sintesis en la que se produce la explicación del hecho y se da el valor a los datos aportados por la investigación. Sin embargo, el historiador se mueve hoy por un campo más complejo en el que necesita la colaboración interdisciplinar, al igual que él participa de todas las demás disciplinas" ${ }^{20}$.

Por lo que se refiere a las fuentes, para la Historia Antigua son todo aquello que pueda aportar información sobre las cuestiones de estudio. Una perspectiva crítica, cuasi objetiva, y las relaciones con otras disciplinas favorecerán otros enfoques, camino a la obtención de respuestas. 
Ya que lo que pretendo es estudiar y analizar ciertas cuestiones de esta civilización desde su propio punto de vista, no desde otras perspectivas alejadas de los acontecimientos que pudieron manipular la información, se recurrirá como fuentes a las arqueológicas y artísticas, como la arquitectura (funeraria, religiosa, residencial, etc.), la escultura ${ }^{21}$, los relieves, pinturas y jeroglíficos ${ }^{22}$, las piezas de joyería, mobiliario, etc. de los ajuares funerarios, amuletos, sellos, escarabeos, etc., la cerámica o los objetos de uso cotidiano. Pero, además de este tipo de fuentes, también han de tomarse en cuenta las epigráficas (Piedra de Palermo, estelas, inscripciones regias, biografías de funcionarios, etc.) y papirológicas (como el Papiro Real de Turín, el Papiro Westcar y el papiro Ebers, entre otros).

Tal diversidad y extensión de fuentes es precisa para extraer una documentación completa, que abarque documentación cronológica, administrativa, social, política, artística, religiosa, literaria,...

Además, se consultarán los trabajos de investigadores anteriores que aludan o traten, en cierta medida, el tema que nos concierne, confrontando opiniones o siguiendo líneas o ideas ya marcadas como guía.

Es esencial tener claro que toda la información histórica extraíble de esta gran cantidad de fuentes no puede ser considerada directamente como real e histórica, sino que "deben de ser cuestionadas en función de su esquema mental: qué seleccionan y qué orden de los acontecimientos establecen, es decir, en función de su imagen histórica (...), imagen ritual sustraída a las categorías corrientes, excesivamente esquemáticas, cíclico/lineal (...) la historia era para el egipcio una renovación festiva de acontecimientos originarios y se celebraba (...) según un ritual"23.

\section{CONCLUSIONES}

En mis lecturas iniciales he podido comprobar que, inversamente a lo que sucede en estudios de otras sociedades antiguas, hay un conocimiento más amplio sobre la situación de la mujer en los estratos más bajos de la sociedad, donde se está comprobando, como dije antes, la existencia de una situación de igualdad, mientras que se mantiene la imagen tradicional de la mujer en la realeza. El rey es el que manda y ella es una figura dependiente de él, sin poder, ni voz, ni voto, simplemente apaciguadora del dios, para

que todo transcurra de forma adecuada durante el reinado de su marido; pero, si accede al trono, se contempla como usurpadora o mera regente, potencialmente manipulable, y su gobierno es calificado negativamente, como por ejemplo Hatshepsut (aunque, desde hace años, hay abundantes detrac- 
tores de la imagen peyorativa de esta reina). Otra idea de gran difusión, presente en algunos círculos de investigación, es que la importancia de las mujeres y su poder se basaban en que ellas legitimaban el ascenso al trono, por vía de la maternidad. ¿En base a qué criterios no experimentaron la misma situación de igualdad que las mujeres del resto de la sociedad?

Otras cuestiones, que están siendo revisadas por investigaciones actuales, son la poligamia en la realeza y la presencia de barenes $^{24}$.

Así pues, aún queda mucho camino por transitar y, desde mi posición de joven investigadora, espero contribuir con mi labor en el quehacer histórico con el fin de lograr una historia completa, que abarque a ambas mitades de la humanidad.

\section{BIBLIOGRAFÍA}

BAGNALL, R. S.; CRIBIORE, R., Women's letters from ancient Egypt: 300 $B C-A D$ 800, Michigan University Press, 2006.

BAINES, J.; MÁLEK, J., (trad. Thema, S.A.), Atlas culturales del mundo. Cultura y sociedad del Antiguo Egipto, Barcelona: Ediciones Folio, 2002. 2007.

BEDMAN, T., Reinas de Egipto. El secreto del poder, Madrid: Alianza Ed.,

BOCK, G., "La historia de las mujeres y la historia de género: Aspectos de un debate internacional, Historia Social, 9, España: Universidad de Valencia, Instituto de Historia Social, 1991, págs. 55-77.

CASADO QUINTANILLA, B. (coord.), Tendencias historiográficas actuales, Madrid: UNED, 2004.

COMESAÑA SANTALICES, G. M., "La ineludible metodología de Género", Revista Venezolana de Ciencias Sociales, vol. 8, n 1, de Enero-Junio, 2004, en http:/ /web.uvigo.es/pmayobre

DESROCHES NOBLECOURT, Ch., La mujer en tiempos de los faraones, Madrid: Editorial Complutense, 1999.

FISCHER, H. G., Egyptian Women of the Old Kingdom and of the Heracleopolitan Period, Nueva York: The Metropolitan museum of Art, 1989.

GARDINER, A. H., "A New Masterpiece of Egyptian Sculpture”, Journal of Egyptian Archaeology, 4, 1917, págs. 1-3.

GUGEL, B., “¿Harenes y concubinas en el antiguo Egipto?”, Boletin de la Asociación Española de Egiptología, no 15, 2005, págs. 7-28.

HAWASS, Z., Silent Images: Women in Pharaonic Egypt, Estados Unidos: 
Abrams, 2000.

HORNUNG, E. (Trad. F. Ballesteros Balbastre), Introducción a la Egiptología. Estado, métodos, tareas, Madrid: Ed. Trotta, 2000.

LESKO, B. S., Women and Religion in Ancient Egypt, Brown University, 2002, en: www.stoa.org/diotima

PESTMAN, P. W., Marriage and Matrimonial Property in Ancient Egypt: A contribution to establishing the legal position of the woman, Papyrologica LugdunoBatava Vol. 9, Leiden: Brill, 1961. Press, 1993.

ROBINS, G., Women in ancient Egypt, Gran Bretaña: Harvard University

SCHULZ, R.; SEIDEL, M. (eds.), Egipto. El mundo de los faraones, Königswinter: Könemann, 2004.

SWEENEY, D., Correspondence and Dialogue: Pragmatic Features in Late Ramesside Letter-writing (Ägypten und Altes Testament 49), Wiesbaden: Harrassowitz, 2001.

TROY, L., "Good and Bad Women. Maxim 18/284-288 of the Instructions of Ptahhotep”, en GM 80, 1984, 77-81.

TROY, L., Patterns of Queenship in Ancient Egyptian Myth and History, Uppsala: Almquist \& Wiksell International, 1986.

VERCOUTTER, J., "La Femme en Égypte ancienne”, en Histoire Mondiale de la Femme, I, 1965, pp.61-152.

WARD, W. A., "The egyptian economy and non-royal women: their status in public life", NEH Lecture, Brown University, 21 June, 1995, en www.stoa.org/diotima

WARD, W. A., Essays on Femenine Titles of the Middle Kingdom and Related Subjects,Beirut, American University of Beirut: Syracuse University Press, 1986.

\section{Notas}

${ }^{1}$ Producto de la cual será la Egiptología tal como la conocemos actualmente.

${ }^{2}$ En la dirección del Servicio de Antigüedades, Gaston Maspero (1846-1916) sucederá a Mariette.

${ }^{3}$ En 1925 G. A. Reisner descubrió la tumba de Hetep-heres I, reina de la IV dinastía y madre del faraón Keops, con un espléndido ajuar funerario en el que destacan las joyas y el mobiliario. 
${ }^{4}$ BAINES, J.; MÁLEK, J., Atlas culturales del mundo. Cultura y sociedad del Antiguo Egipto, Barcelona: Ediciones Folio, (trad. Thema, S.A.), 2002, p. 29.

${ }^{5}$ A pesar de todo, la Egiptología en España no cuenta con una suficiente presencia ni académica ni institucionalmente.

6 "' $\mathrm{Si}$, como parece, en los textos egipcios se encuentra casi solo una lengua métricamente conformada, no deberíamos servirnos de un concepto de 'literatura' demasiado estrecho, sino que, junto a las obras teológicas, políticas y científicas, convendría incluir también cartas y biografías. La denominación egipcia de 'escritos' o 'escritos de los antepasados' (...), parece ser aplicable a todas las manifestaciones escritas actuales de los 'antepasados', incluso a sus actos y listas; ciertamente, las cuestiones propiamente terminológicas aún no han sido suficientemente investigadas" [HORNUNG, E. (Trad. Francesc ballesteros Balbastre), Introducción a la Egiptología. Estado, métodos, tareas, Madrid: Ed. Trotta, 2000, p. 51].

${ }^{7}$ BOCK, Gisela, "La historia de las mujeres y la historia de género: Aspectos de un debate internacional, Historia Social, 9, España: Universidad de Valencia, Instituto de Historia Social, 1991, p. 56.

${ }^{8}$ Ibidem., p. 60.

'COMESAÑA SANTALICES, G. M., "La ineludible metodología de Género", Revista Venezolana de Ciencias Sociales, vol. 8, no 1, de Enero-Junio, 2004, en http://web.uvigo.es/pmayobre (acceso: 22/12/2009), p. 11.

${ }^{10}$ GILLIGAN, C., In a different voice: Psychological theory and women's development, Cambridge, MA: Harvard University Press, 1982.

${ }^{11}$ BOCK, G., "La historia de las mujeres...", op. cit., p. 56.

${ }^{12}$ Ibidem,p.60.

${ }^{13}$ Véase bibliografía.

${ }^{14}$ Ibidem, p. 57.

${ }^{15}$ Ibidem., p. 58.

${ }^{16}$ WARD, W. A., "The egyptian economy and non-royal women: their status in public life", NEH Lecture, Brown University, 21 June, 1995, en www.stoa.org/diotima

${ }^{17}$ Documentado en los denominados Textos de las pirámides y en los Textos de los sarrófagos, estudiados, entre otros, por R. O. Faulkner (The Ancient Egyptian Pyramid Texts, Oxford, 1969; The Ancient Egyptian Coffin Texts, III Vols., Warminster, 1973- 1978).

18 “Antigua diosa de la caza y de la guerra a la que se adoró en el Imperio Antiguo en la región menfita como protectora de la monarquía. Pero su lugar principal de culto era Sais, en el Delta (...). Posteriormente avanzaría en Esna hasta el rango de divinidad de la creación. Neith portaba la corona del bajo Egipto y su símbolo es un escudo con flechas cruzadas" [SCHULZ, R.; SEIDEL, M. (eds.), Egipto. El mundo de los faraones, Königswinter: Könemann, 2004, p. 523]. 
${ }^{19}$ Sobre el Dinástico Temprano (c. 3000- 2686 a.n.e.) y el Imperio Antiguo (2686-2125 a.n.e.) versará mi Trabajo Fin de Máster, actualmente en preparación.

${ }^{20}$ CASADO QUINTANILLA, B. (coord.), Tendencias historiográficas actuales, Madrid: UNED, 2004, p. 166.

${ }^{21}$ La escultura era una forma de conservación de la personalidad física y espiritual del muerto, se trataba de una estatua "animada". El escultor en el Antiguo Egipto era denominado el "vivificador", y daba vida a la escultura mediante el ritual de "apertura de la boca". Otras de las formas de alojamiento eran: la momia, la estela real, la pirámide y el monumento funerario.

22 Aunque no son considerados artísticamente, pertenecen a los bajorrelieves.

${ }^{23}$ HORNUNG, E. (Trad. Francesc ballesteros Balbastre), Introducción a la..., op. cit., p. 127.

${ }^{24}$ GUGEL, B. "¿Harenes y concubinas en el antiguo Egipto?”, Boletin de la Asociación Española de Egiptología, no 15,2005 , pp. 7-28. 